\title{
Multiexciton Spectroscopy of a Single Self-Assembled Quantum Dot
}

\author{
E. Dekel, ${ }^{1}$ D. Gershoni, ${ }^{1}$ E. Ehrenfreund, ${ }^{1}$ D. Spektor, ${ }^{1}$ J. M. Garcia, ${ }^{2}$ and P. M. Petroff ${ }^{2}$ \\ ${ }^{1}$ Physics Department and Solid State Institute, Technion-Israel Institute of Technology, Haifa, 32000, Israel \\ ${ }^{2}$ Materials Department, University of California, Santa Barbara, California 93106
}

(Received 28 January 1998)

\begin{abstract}
We apply low temperature confocal optical microscopy to spatially resolve, and spectroscopically study, a single self-assembled quantum dot. By comparing the emission spectra obtained at various excitation levels to a theoretical many body model, we show that (a) single exciton radiative recombination is very weak, and (b) sharp spectral lines are due to optical transitions between confined multiexcitonic states among which excitons thermalize within their lifetimes. Once these few states are fully occupied, broadbands appear due to transitions between states which contain electrons in the continuum. [S0031-9007(98)06202-4]
\end{abstract}

PACS numbers: 78.66.Fd, 71.35.-y, 71.45.Gm, 85.30.Vw

The study of electronic processes in semiconductor heterostructures of reduced dimensionality has been a subject of recent extensive research efforts. Of particular importance are the efforts to fabricate and study semiconductor quantum dots (QDs) of nanometer size, in which the charge carriers are confined in all directions to characteristic lengths which are smaller than their de Broglie wavelengths [1-16]. These efforts are motivated by both the QDs potential device applications, as well as their being an excellent stage for experimental studies of basic quantum mechanical principles. One very promising system of such QDs is called self-assembled QDs (SAQDs). In fabricating SAQDs, minimization of the lattice mismatch strain between different epitaxially grown semiconductor layers occurs via the formation of small islands connected by a very thin wetting layer. By capping these self-assembled islands with an epitaxial layer of wider band gap material, with similar lattice constant to that of the substrate, high quality QDs are produced $[4,5]$. This natural way of producing large ensembles of QDs has motivated a vast number of studies of their structural, electronic, and optical properties [3-5,10-16]. The size distribution of these SAQDs (typically about 10\%), and the resultant inhomogeneous broadening of the SAQDs characteristic features, has so far limited the ability to clearly understand and unambiguously interpret the experimental results. In this Letter, we overcome this obstacle by spectroscopically studying multiexcitonic optical transitions in a single SAQD. We show here, indeed, that multiple sharp spectral lines, as well as broad spectral features, which previously were interpreted as an optical signature for emission from an ensemble of dots [4,5], are actually due to optical transitions between multicarrier states within a single dot, under various excitation levels.

The SAQD sample studied here was fabricated by deposition of a coherently strained epitaxial layer of InAs on an AlGaAs layer deposited on GaAs substrate. The layer sequence, compositions, and widths are given in the left inset of Fig. 1. During the growth of the strained layer, the sample was not rotated; thus a gradient in the QDs density was formed across its surface [10]. In particular, low density areas, in which the average distance between neighboring QDs is larger than our spatial resolution, could easily be found on the sample surface. The right side inset of Fig. 1 displays the far-field ensemble photoluminescence (PL) spectrum of such an area of the SAQD sample.

We use a $\times 100$ in situ microscope objective for diffraction limited low temperature confocal optical

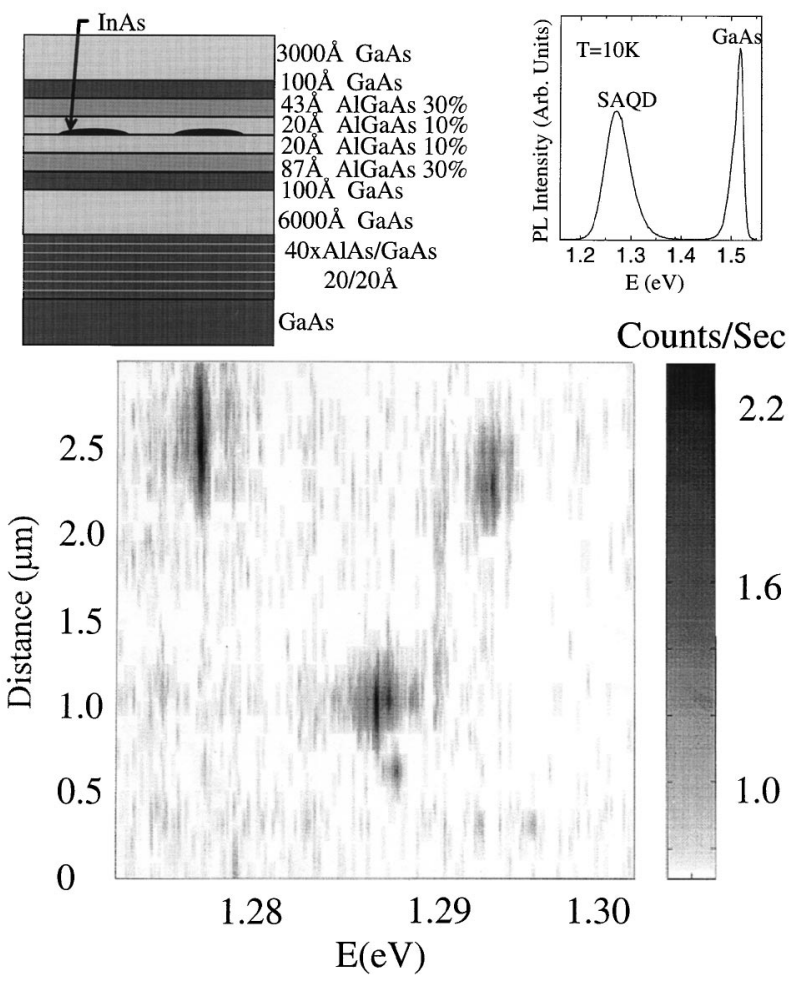

FIG. 1. PL intensity as a function of photon energy and position along a line across the SAQD sample surface. The intensity is given by a gray color scale bar to the right. Left inset: schematic description of the sample. Right inset: far-field PL spectrum of the sample. 
spectroscopical studies of the single SAQDs. Our system provides spatial resolution of $\simeq 0.5 \mu \mathrm{m}$, both in the excitation and in the detection channels [14].

The dots position and characteristic emission wavelength are found by taking PL spectra during a line scan over the SAQD sample surface. A typical scan is displayed in Fig. 1, where the PL intensity as a function of photon energy and objective position is given by the gray color scale. During this scan, the PL was excited with a $730 \mathrm{~nm}, 7 \mu \mathrm{W}$ $\mathrm{cw}$ light from a Ti:S laser. In each $0.1 \mu \mathrm{m}$ long step of the objective the PL spectrum was measured by exposing a cooled charge coupled device camera for $50 \mathrm{sec}$. Three emission lines from three different spatial positions along the scanned line are evident in Fig. 1. These lines are due to carriers' recombination within single SAQDs, as indicated by their spatial and spectral widths, which are both resolution limited [6,17]. In Fig. 2 we present PL spectra from a single SAQD found by such a scan for various excitation powers.

An overall perception of the important spectral features is obtained from the $100 \mu \mathrm{W}$ spectrum which is composed of two groups of emission lines located near 1.325 and $1.375 \mathrm{eV}$, respectively. The groups are nearly symmetrically positioned around a weak emission line at $1.355 \mathrm{eV}$. Each group is composed of several sharp and well resolved lines, two of which, roughly $7 \mathrm{meV}$ apart, are particularly strong. We marked the strongest lines in each group and the center line by numbers in increasing order of their spectral position. It is important to note here that the overall shape of the spectrum and its power dependence (see below) are quite typical to all of the dots we studied. However, as can be deduced from the far-field

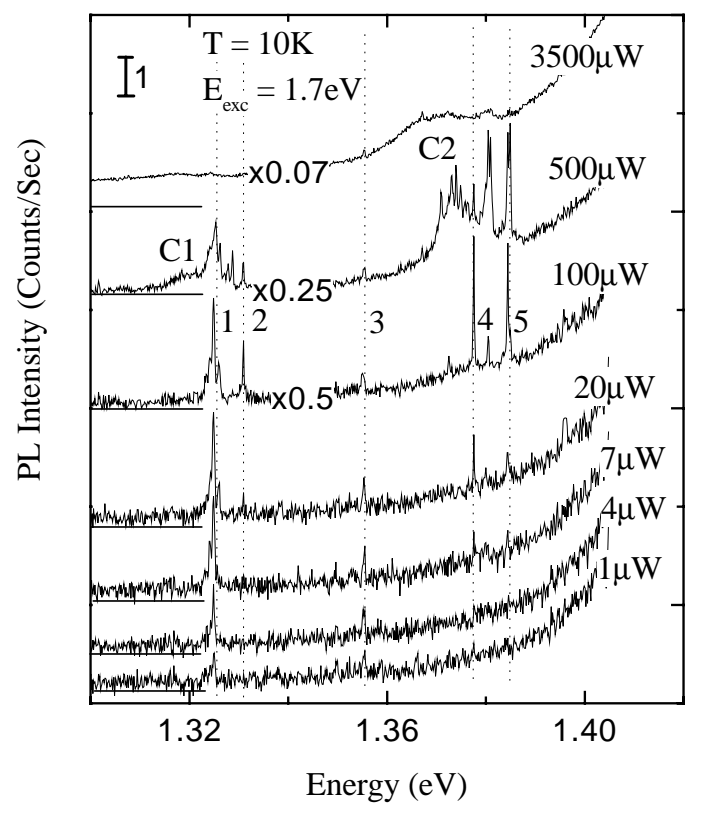

FIG. 2. PL spectra from a single SAQD for various excitation power levels.
PL spectrum (right side inset of Fig. 1) different PL spectra are obtained from different dots, depending on their dimension, shape, composition, and surroundings. At the excitation power of $1 \mu \mathrm{W}$, only lines 1 and 3 (Fig. 2) are observed, with intensity of less than 1 count/s, in agreement with the estimated exciton lifetime [11] and our system's collection efficiency. The emission of line 1 increases roughly as the square root of the excitation power up to about $100 \mu \mathrm{W}$, where it reaches saturation. Line 3, on the other hand, reaches saturation already at excitation power of $7 \mu \mathrm{W}$. At $20 \mu \mathrm{W}$ the emission spectrum is already composed of the five main spectral lines, and at $100 \mu \mathrm{W}$, all main lines are saturated. At saturation, the intensity of line 2 is about half that of lines 1 , 4 , and 5 , whose intensity is $\simeq 10$ times larger than that of line 3. Above $100 \mu \mathrm{W}$, several sharp lines appear below each group of lines. At yet higher excitation power, these lines form two broad spectral bands ( $C 1$ and $C 2$, Fig. 2) which dominate the PL spectrum.

For the analysis of our observations we use a model parallelepipedal box with infinite potential barriers and a rectangular base whose dimensions are much larger than its height. We fitted the dot base dimensions to obtain the observed level separation of $\simeq 50 \mathrm{meV}$ and adjusted its height to obtain the Coulomb splitting of $7 \mathrm{meV}$ (see below). Using typical InAs effective masses [18] for electrons $\left(0.023 m_{0}\right)$ and for heavy holes $\left(0.6 m_{0}\right.$, where $m_{0}$ is the electron rest mass) and dielectric constant [18] $(\varepsilon=15)$ we find that base dimensions of $30.2 \times$ $31.2 \mathrm{~nm}^{2}$ and a height of $5 \mathrm{~nm}$ best fit the observed data. Note that the box base is not square; thus there is no geometrical degeneracy in agreement with recent calculations [15]. Although our model does not describe the exact SAQD potential structure in geometrical shape [5], strain, and piezoelectric fields [15,16], it is reassuring that the fitted dimensions are similar to those typically reported for this SAQD system [5]. We show below that in spite of its simplicity the model explains our data quite well. This means that the knowledge of the single particle level separation and the strength of the Coulomb matrix elements between these levels are nearly enough to describe the optical properties of a fully quantized system with a few carriers in it. The details of its confining potentials are only second in importance.

We consider the first four, doubly degenerate electron and hole levels in our model dot: (111), (121), (211), and (221) at this energy order, where the numbers in parentheses are the quantum numbers associated with the confinement along $x, y$, and $z$ (the growth direction), respectively. The wave functions of electrons and holes in these states are analytically expressed. This limited number of states is adequate to explain the low level excitation PL spectra. Higher excitation levels, which give rise to carriers in the continuum above the dot barriers' potential, are dealt with here more qualitatively. The Coulomb interaction between carriers in our dot is now 
considered using the following many body Hamiltonian: $H=H_{\text {free }}+H_{\text {Coul }}$, where $H_{\text {free }}$ describes noninteracting electrons $(e)$ and holes $(h)$ in their respective bands, and $H_{\text {Coul }}$ describes the $e-e, h-h$, and $e-h$ Coulomb direct and exchange interactions [19]. Since electron and hole wave functions are identical in our model, the $e-h$ exchange interaction term vanishes. We find the solution for the multiexciton energy levels and wave functions, by exact diagonalization of the many body Hamiltonian for these first four single carrier levels. We consider here all neutral multiexcitonic states up to the exciton population number of eight, at which all of the levels considered are fully occupied. Optical transitions between the different excitonic population levels, in which a single $e-h$ pair is annihilated, are then calculated using the dipole approximation [19].

In Fig. 3, horizontal solid lines represent some of the calculated energy levels. The single carrier states (for which the Coulomb interaction is ignored) are displayed on the left side of the figure. Single electron (hole) energy levels are represented by superscripts above the letters $e(h)$ and the occupation number of these levels is given by

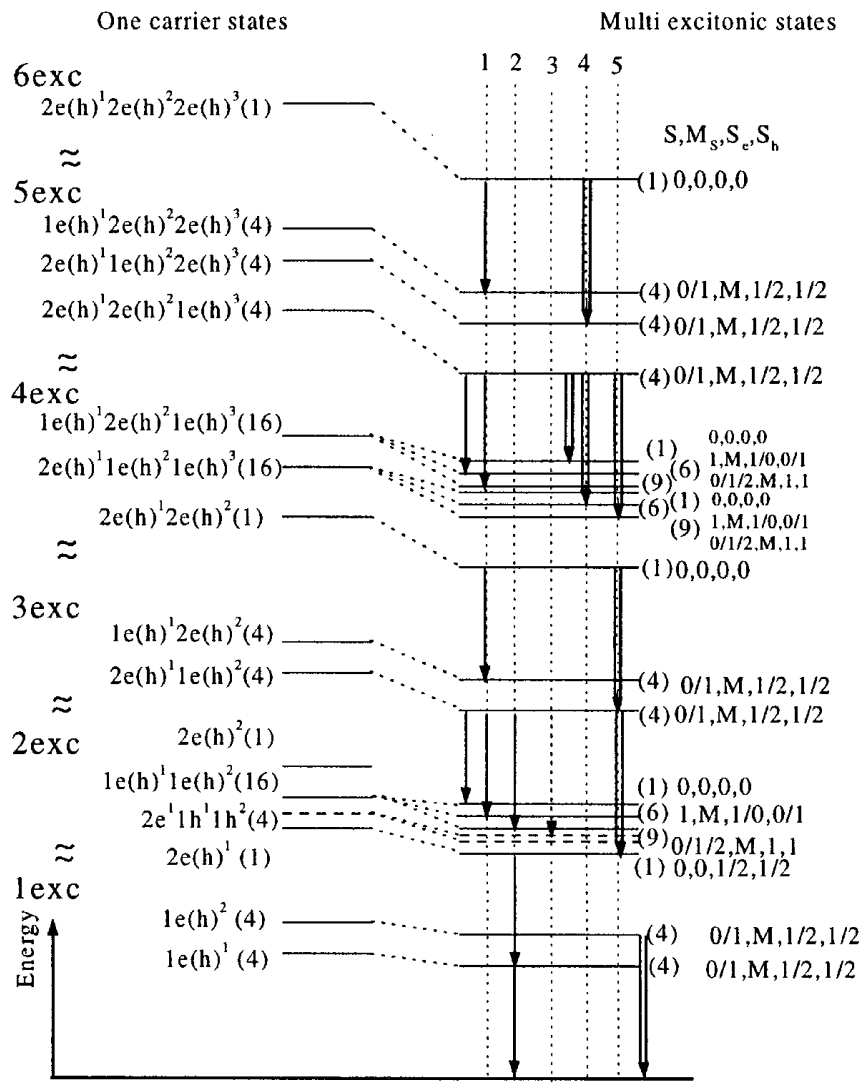

FIG. 3. Energy level diagram of the SAQD multiexcitonic states, contributing the most to optical emission. Shown are the multiexcitonic levels calculated by excluding (left) and including (right) the Coulomb interaction between carriers. The vertical arrows indicate optical transitions due to one exciton annihilation (see text). numbers in front of the letter. The state spin degeneracy is given by the number in parentheses. The multiexcitonic states, including now the Coulomb interaction between the carriers, are displayed on the right side of the figure. Here, the degeneracy is partially removed and we describe the states by their total angular momentum $(S)$, its projection on the dots growth direction $\left(M_{S}\right)$, and the total electronic $\left(S_{e}\right)$ and hole $\left(S_{h}\right)$ spins. With one exception, indicated by dashed horizontal lines, we display only those states which evolve from single carrier levels of identical electron and hole quantum and occupation numbers. This is because the lowest energy state in each excitonic occupation level is always of this type, and thus, optical transitions between these levels dominate the PL spectrum. These single carrier states are either 1, 4, or 16 times degenerate. The Coulomb interaction lifts only the degeneracy of the 16 multiplets, which occur whenever two half filled levels of electron and hole participate in the multiexcitonic state. They are split into three levels according to their $S_{e}$ and $S_{h}$ quantum numbers, exactly like a bulk biexciton [20]. The lowest of these levels is ninefold degenerate containing a quintuplet, a triplet, and a singlet of $S_{e}=1$ and $S_{h}=1$ which add to $S=2,1$, and 0 , respectively. The midenergy level is sixfold degenerate containing two triplets of $S_{e}=1(0)$ and $S_{h}=0(1)$ which add to $S=$ 1(1). The highest of these levels contains only a singlet with $S_{e}=0, S_{h}=0$, and $S=0$. For calculating the PL spectrum the distribution of excitons among their multiexcitonic states should be known. The relatively small number of observed PL lines lead us to safely conclude that only the lowest multiexcitonic energy state of each exciton occupation number has a significant steady state population. This means that there is no phonon bottleneck [21] for exciton thermalization and they reach thermal distribution faster than the radiative recombination occurs.

The optically allowed transitions between the lowest multiexcitonic state of each exciton occupation level and the multiexcitonic states of one less exciton occupation are represented in Fig. 3 by vertical arrows. Annihilations of $e^{1} h^{1}\left(e^{2} h^{2}\right)$ are represented by solid (empty) arrows, and annihilation of $e^{2} h^{1}$ is represented by a dashed arrow. We note that optical transitions conserve $S$ and $M_{S}$ [19]. Transitions of the same energy between different exciton occupation levels are drawn along the same vertical thin dashed line. These vertical lines are numbered in increasing order of their energies, like the experimentally measured PL lines as shown in Fig. 4. In Fig. 4 we display the calculated dot emission spectra, in units of the squared dipole moment between the $e^{1}$ and $h^{1}$ states, for a few exciton occupation numbers. In the calculations, we average over degenerate initial states and sum over final states. For comparison, measured spectra for various excitation powers are shown by dots.

The following conclusions are drawn by comparing the experimental data to the model calculations. The 


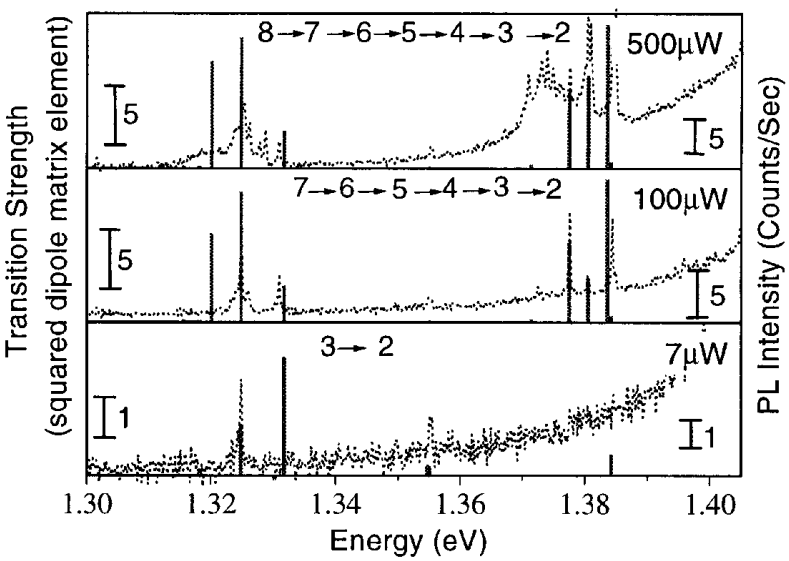

FIG. 4. Calculated PL spectra for various exciton population numbers. For comparison, the experimental measurements are also displayed.

characteristic appearance of the PL lines in pairs is clearly explained in terms of the Coulomb splitting of biexciton levels (the nine and six degenerate levels). The multiline PL spectrum which we measure even at our lowest excitation power indicates that the average exciton occupation number is already larger than 2 . This agrees very well with a conservative estimate of the occupation number, based on the measured laser power and estimations of the absorption and exciton lifetime [11] (diffusion of carriers from the AlGaAs layers into the SAQD can be safely neglected due to the sample structure). Thus, surprisingly, single lines due to the recombination of single exciton and single biexciton are not observed at all at low excitation spectra. Line 2, which corresponds in energy to the radiative recombination of a single exciton, appears only at much higher excitation power $(20 \mu \mathrm{W})$. This can be due to $e-h$ exchange, which splits the fourfold degeneracy between the triplet and the singlet states of the single exciton level. Since, as has been observed in II-VI nanocrystallites [8], the lower energy triplet state is optically forbidden, the single exciton annihilation line can be observed only when at least three excitons occupy the dot. Alternatively, it can be due to a reduced electron-hole overlap integral which weakens this transition [15].

Assuming that recombination is possible only via radiative channels we calculated the lifetime of each multiexcitonic occupation level. Using these lifetimes in turn, a correlation between the excitation power and the number of excitons within the dots can be drawn. We find a relatively good agreement between the excitation power dependence of the PL spectra and the calculated spectra based on that correlation. The appearance of sharp lines at the lower energy side of each of the two spectral line groups, and their evolution with increasing excitation power into the broadbands $C 1$ and $C 2$, are also explained by our model (see the $500 \mu \mathrm{W}$ PL spectrum in Fig. 4). We note in Fig. 3 that the higher the excitation is, the higher are the populated single electron and hole levels which participate in the multiexcitonic ground states. Optical transitions from these states to the high energy states of one less exciton level by the annihilation of $e^{1} h^{1}$ and $e^{2} h^{2}$ are now possible, leading to a characteristic decrease in the energy of these transitions. The magnitude of this shift is given by the Coulomb interaction term between the high energy level and the lower one. This term is almost constant as long as confined single carrier states are concerned. At high enough excitation power, when continuum electron levels are populated, these shifts are becoming smaller and thus, broad spectral bands at low energy are formed. Since the density of continuum levels is large, these bands are not saturated, and they eventually dominate the PL spectra. We do not observe higher energy PL lines due to the annihilation of higher energy $e$ - $h$ pairs, such as $e^{3} h^{3}$ and $e^{4} h^{4}$, probably since, as can be seen in Fig. 2, they are masked by the large PL emission from the GaAs substrate.

In summary, we resolved the emission from a single self-assembled quantum dot and successfully explained its power dependent PL spectra using multicarrier Hamiltonian.

[1] M. A. Reed et al., Phys. Rev. Lett. 60, 535 (1988).

[2] K. Brunner et al., Phys. Rev. Lett. 69, 3216 (1992); 73, 1138 (1994).

[3] C. W. Sneyder et al., Phys. Rev. Lett. 66, 3032 (1991).

[4] J.-Y. Marzin et al., Phys. Rev. Lett. 73, 716 (1994).

[5] H. Drexler et al., Phys. Rev. Lett. 73, 2252 (1994).

[6] D. Gammon et al., Phys. Rev. Lett. 76, 3005 (1996).

[7] U. Bockelmann et al., Phys. Rev. Lett. 76, 3622 (1996).

[8] M. Nirmal et al., Phys. Rev. Lett. 75, 3728 (1995).

[9] W. Wegscheider et al., Phys. Rev. Lett. 79, 1917 (1997).

[10] S. Fafard et al., Phys. Rev. B 52, 5752 (1995).

[11] S. Raymond et al., Phys. Rev. B 54, 11548 (1996).

[12] M. Grundmann et al., Phys. Rev. Lett. 74, 4043 (1995).

[13] V. A. Shchukin et al., Phys. Rev. Lett. 75, 2968 (1995).

[14] E. Dekel et al., Physica (Amsterdam) E (to be published).

[15] J. Kim et al., Phys. Rev. B 57, 9408 (1998).

[16] C. Pryor et al., Phys. Rev. B 56, 10404 (1997).

[17] H. F. Hess et al., Science 264, 1740 (1994).

[18] S. Adachi, J. Appl. Phys. 58, R1 (1985).

[19] A. Barenco and M. A. Dupertuis, Phys. Rev. B 52, 2766 (1995).

[20] J. J. Forney et al., Nuovo Cimento B 22, 153 (1974).

[21] H. Benisty, Phys. Rev. B 51, 13281 (1995). 